Revue d'histoire de l'Amérique française

REVUE D.HISTOIRE DE L'AMÉRIQUE FRANÇAISE

\title{
Introduction : la Nouvelle-France et l'Atlantique
}

\section{Catherine Desbarats et Thomas Wien}

Volume 64, numéro 3-4, hiver-printemps 2011

La Nouvelle-France et l'Atlantique

URI : https://id.erudit.org/iderudit/1017968ar

DOI : https://doi.org/10.7202/1017968ar

Aller au sommaire du numéro

\section{Éditeur(s)}

Institut d'histoire de l'Amérique française

\section{ISSN}

0035-2357 (imprimé)

1492-1383 (numérique)

Découvrir la revue

\section{Citer ce document}

Desbarats, C. \& Wien, T. (2011). Introduction : la Nouvelle-France et l'Atlantique. Revue d'histoire de l'Amérique française, 64(3-4), 5-29. https://doi.org/10.7202/1017968ar d'utilisation que vous pouvez consulter en ligne.

https://apropos.erudit.org/fr/usagers/politique-dutilisation/ 


\title{
Introduction : La Nouvelle-France et l'Atlantique ${ }^{\mathrm{I}}$
}

\author{
Catherine Desbarats \\ Université McGill
}

THOMAS WiEN

Université de Montréal

Dort-Royal et Québec, Plaisance et La Mobile (Fort Louis de la
Louisiane), Louisbourg et La Nouvelle-Orléans - ces paires de toponymes désignent des pôles successifs de l'implantation française en Amérique septentrionale aux XVII ${ }^{\mathrm{e}}$ et XVIII ${ }^{\mathrm{e}}$ siècles. Les évoquer, c'est immobiliser provisoirement, sur la carte de cette Amérique encore largement amérindienne, une Nouvelle-France au sens étendu du terme ${ }^{2}$. Pour expliquer les «déplacements » de l'ensemble, l’histoire a longtemps privilégié, comment pouvait-il en être autrement, l'action des colonisateurs et des colons. Elle a insisté, plus précisément, sur le jeu des puissances européennes rivales - l'Acadie perdue, la Louisiane gagnée, etc. - et le dynamisme des Français d’Amérique, les Canadiens en tête. Elle insérait

1. Nous remercions l'ensemble des auteurs et des évaluateurs anonymes de ce numéro spécial ainsi que les participants au colloque "Mondes atlantiques français» qui s'est déroulé dans le cadre du $62^{\mathrm{e}}$ congrès annuel de l'IHAF, Montréal, octobre 2009. Nous remercions aussi Robert Gagnon qui nous a généreusement ouvert les pages de la Revue d'histoire de l'Amérique française.

2. Sur ce toponyme nomade et polysémique, voir l'article de C. Desbarats et A. Greer. On aura compris que par «Nouvelle-France», nous retenons, avec une partie des contemporains et historiens, une acception large, la zone de colonisation ou d'influence française qui s'étendrait finalement d'un golfe à l'autre. Il s'agit donc de l'«Amérique française» survolée récemment par Gilles Havard et Cécile Vidal dans Histoire de l'Amérique française (Paris, Flammarion, coll. "Champs », 2006 [2003]), deuxième édition. Un autre usage contemporain repris lui aussi par des historiens la restreint à une zone plus nordique, excluant la Basse-Louisiane. 
ce mouvement globalement expansionniste dans un schéma plus général dont les deux phases se chevauchaient: le déploiement colonial en terre américaine et l'émergence de peuples créoles appelés à gagner en autonomie et, si les astres étaient bien alignés, à accéder à l’indépendance. L'histoire coloniale trouvait là son substrat métahistorique. À l'enracinement progressif des colons correspondait leur éloignement progressif de l'Europe, les deux phénomènes étant inscrits dans le cours «naturel» de l'histoire ${ }^{3}$.

Ce n'est pas depuis hier que les bases de ce récit téléologique, en symbiose avec le développement de l'État-nation du XIX ${ }^{\mathrm{e}}$ siècle et au-delà, ont été minées, pour l'ensemble des Amériques d'ailleurs. Minées par des sensibilités changeantes, sans doute, mais aussi par de nouvelles manières d'écrire l'histoire coloniale ${ }^{5}$. L'histoire sociale qui vers 1970 s'est intéressée aux existences individuelles cachées par les archétypes populaires, a insisté sur les clivages au sein du peuple créole. C’était dépeindre une entreprise coloniale déjà plus compliquée. Et l'élan d'inclusivité de cette histoire favorisait à la longue l'élargissement de l'enquête aux Amérindiens et Africains qui, esclaves, domiciliés ou libres, fréquentaient de près les colons dans leurs principaux établissements. L'histoire traditionnelle les avait pourtant marginalisés ${ }^{6}$.

3. Quelques références: Serge Gagnon, Le Québec et ses historiens de 1840 à 1920. La Nouvelle-France de Garneau à Groulx (Québec, Les Presses de l’Université Laval, 1978); Bruce G. Trigger, Les Indiens, la fourrure et les Blancs. Français et Amérindiens en Amérique du Nord (Montréal/Paris, Boréal/Seuil, 1990), 11-72 ; JeanFrançois Mouhot, «L'influence amérindienne sur la société en Nouvelle-France: une exploration de l'historiographie canadienne de François-Xavier Garneau à Allan Greer (1845-1997)", Globe. Revue internationale d'études québécoises, 5,1 (2002): 123-157; Joyce Chaplin, "Expansion and Exceptionalism in Early American History ", Journal of American History, 89,4 (2003): 1431-1455 ; Jack P. Greene, "Colonial History and National History: Reflections on a Continuing Problem ", William and Mary Quarterly, 64,2 (2007): 235-250.

4. Quelques échantillons de l'historiographie récente portant sur d'autres parties de l'Amérique du Nord coloniale ou adoptant une perspective comparative: Daniel Richter, Before the Revolution: America's Ancient Pasts (Cambridge, Harvard University Press, 2011); Alan Taylor, American Colonies (New York, Viking/Penguin, 2001); Stephanie Gail Wood, Transcending Conquest: Nahua Views of Spanish Colonial Mexico (Norman, University of Oklahoma Press, 2003); Paul Mapp, The Elusive West and the Contest for Empire, 1713-1763 (Chapel Hill, University of North Carolina Press, 2011); Lauren Benton, A Search for Sovereignty: Law and Geography in European Empires, 1400-1900 (New York, Cambridge University Press, 2010); Eric Hinderaker et Rebecca Horn, "Territorial Crossings: Histories and Historiographies of the Early Americas », William and Mary Quarterly, 67,3 (2010): 395-432.

5. On comprendra que ce n'est pas la question nationale comme telle qui est visée ici, mais bien l'incidence des nationalismes sur la manière d'écrire l'histoire coloniale.

6. Une liste très partielle de travaux d'histoire sociale des années 1970-1980: Louise Dechêne, Habitants et marchands de Montréal au XVII siècle (Paris, Plon, 1974); A. J. B. Johnston, Religion in Life at Louisbourg, 1713-1758 (Montréal/Kingston, McGill-Queen’s University Press), 1984; Carl J. Ekberg, Colonial Ste. Geneviève: An Adventure on the Mississippi Frontier (Gerald, Patrice Press, 1985). Sur les Domiciliés, redécouverts dans les années 1990: Denys Delâge, «Les Iroquois chrétiens des réductions, 1667-1770: 1 
Cette curiosité envers l'Autre se recoupait et se confondait parfois avec celle d'un autre courant de recherche. D'abord liée aux revendications territoriales des Amérindiens des États-Unis au lendemain de la Deuxième Guerre mondiale, l'ethnohistoire s'est peu à peu dotée d'un ensemble de techniques visant à faire parler le non-écrit et à faire parler autrement l'écrit légué par la colonisation ${ }^{7}$. Le but: insérer les premiers peuples dans l'histoire coloniale et, de plus en plus, faire le contraire, c'est-à-dire peser le rôle que la colonisation a joué dans l'histoire amérindienne. De par ses méthodes, ses argumentaires et ses publics, cette histoire demeure polyphonique, par-delà la variété des situations et des trajectoires qui caractérise la mosaïque amérindienne qu'elle a prise sous la loupe ${ }^{8}$. Une impression d'ensemble se dégage néanmoins de ces travaux : jusque dans ses zones d'intense peuplement français, la Nouvelle-France est le cadre d'interactions franco-amérindiennes; dans une bonne partie de ce territoire aux frontières floues, la présence française, loin d'aller de soi, est conditionnelle, à négocier et à renégocier. Privant ainsi la marche de la colonisation de son caractère d'abord perçu comme étant naturel, cette

\footnotetext{
- Migration et rapports avec les Français», Recherches amérindiennes au Québec, 19,1-2 (1991): 59-70, et autres travaux; Marc Jetten, Enclaves amérindiennes. Les "réductions" du Canada, 1637-1701 (Sillery, Septentrion, 1994); Denis Vaugeois, dir., Les Hurons de Lorette (Sillery, Septentrion, 1996); Jan Grabowski, "The Common Ground: Settled Natives and French in Montreal, 1667-1760", thèse de Ph.D. en histoire, Université de Montréal, 1993 ; John A. Dickinson et Jan Grabowski, «Les populations amérindiennes de la vallée laurentienne, 1608-1765", Annales de démographie historique, 85 (1993): 51-65; Daniel H. Usner Jr., "American Indians in Colonial New Orleans", dans Gregory A. Waselkov, Peter H. Wood et Tom Hatley, dir., Powhatan's Mantle: Indians in the Colonial Southeast (Lincoln, University of Nebraska Press, 2006 [1989]), 163-186, deuxième édition; D. H. Usner, Indians, Settlers, and Slaves in a Frontier Exchange Economy (Chapel Hill, University of North Carolina Press, 1992). Sur l'esclavage, avant le renouvellement historiographique des années 1990: Marcel Trudel, L'esclavage au Canada français. Histoire et conditions de l'esclavage (Québec, Les Presses de l'Université Laval, 1960); Mathé Allain, «Slave Policies in French Louisiana », Louisiana History, 21,2 (1980): 127-137 et Carl A. Brasseaux, "The Administration of the Slave Regulations in French Louisiana, 1724-1766», ibid., 139-158, renvoient aux travaux antérieurs sur les esclaves en Louisiane (ou, souvent, sur le traitement des esclaves).

7. B. G. Trigger, Les Indiens..., op. cit., 11-72; du même auteur, «Ethnohistory: The Unfinished Edifice», Ethnohistory, 33,3 (1986): 253-267; Kerwin Lee Klein, Frontiers of Historical Imagination: Narrating the European Conquest of Native America, 1890-1990 (Berkeley, University of California Press, 1997); Patricia K. Galloway, Practicing Ethnohistory: Mining Archives, Hearing Testimony, Constructing Narrative (Lincoln, University of Nebraska Press, 2006); Gilles Havard, «Les Indiens et l'histoire coloniale nord-américaine: les défis de l'ethnohistoire", dans Cécile Vidal et F.J. Ruggiu, Sociétés, colonisation et esclavages dans le monde atlantique. Historiographie des sociétés américaines des XVI ${ }^{e}$-XIX ${ }^{e}$ siècles (Bécherel, Les Perséides, 2009), 95-142.

8. Roland Viau, «Du bon usage de l'ethnohistoire. Essai d'analyse réflexive», dans Roland Tremblay, dir., L'éveilleur et l'ambassadeur. Essais archéologiques et ethnohistoriques en hommage à Charles A. Martijn (Montréal, Recherches amérindiennes au Québec, 1998), 177-186; Philip J. Deloria, «Historiography», dans P. J. Deloria et Neal J. Salisbury, A Companion to American Indian History (Malden, Blackwell, 2002), 6-24; Melissa L. Meyer et K. L. Klein, "Native American Studies and the End of Ethnohistory», dans Russell Thornton, dir., Studying Native America : Problems and Prospects (Madison, University of Wisconsin Press, 1998), 182-216.
} 
histoire modifie aussi la temporalité du récit traditionnel. En soulignant la durable autonomie amérindienne dans la majeure partie de la zone revendiquée par la France, elle incite à mettre de côté le telos de l'écartement des Amérindiens, image-miroir du progrès colonial ${ }^{9}$.

Entre-temps, un autre ensemble hétéroclite de travaux s'est mis à interroger la sphère atlantique, rompant ainsi avec une histoire traditionnelle davantage encline à la traverser. Il nous intéressera davantage ici. Encore une fois, le changement historiographique s'explique en partie par les curiosités de l'histoire coloniale, laquelle en s'intéressant aux migrations transatlantiques, au commerce maritime, ou à la circulation des représentations, se tournait vers l'Europe, voire l'Afrique ${ }^{10}$. Mais là aussi il y a eu rencontre avec ceux qui, parfois depuis fort longtemps, abordaient les colonies nord-américaines de l'extérieur, dans ce cas en étudiant des circulations transatlantiques. Ou avec ceux qui, contre l'arrière-plan de rapprochements nord-atlantiques ou afro-américains, s'intéressaient déjà à des mondes atlantiques ${ }^{11}$. Sur le plan international, une certaine coalescence des différentes approches «atlanticistes» s'est produite vers 1990. L'engouement qui a suivi persiste toujours ${ }^{12}$. L'histoire atlantique demeure

9. Denys Delâge, "L'alliance franco-amérindienne 1660-1701», Recherches amérindiennes au Québec, 19,1 (1989): 1-15; Richard White, Le middle ground. Indiens, empires et républiques dans la région des Grands Lacs, 1650-1815 (Toulouse, Anacharsis, 2010, trad. Frédéric Coton [1991]); Gilles Havard, Empire et métissages. Indiens et Français dans le Pays d'en Haut, 1660-1715 (Sillery/Paris, Septentrion/Presses de l'Université de Paris-Sorbonne, 2003); Kathleen DuVal, The Native Ground: Indians and Colonists in the Heart of the Continent (Philadelphie, University of Pennsylvania Press, 2006).

10. Dale Miquelon, Dugard of Rouen: French Trade to Canada and the West Indies, 1729-1770 (Montréal/ Kingston, McGill-Queen's University Press, 1978); James S. Pritchard, «Ships, Men and Commerce: A Study of Maritime Activity in New France», thèse de Ph.D. en histoire, Université de Toronto, 1973; Jacques Mathieu, Le commerce entre la Nouvelle-France et les Antilles au XVIII siècle (Montréal, Fides, 1981); John G. Clark, New Orleans, 1718-1812: An Economic History (Baton Rouge, Louisiana State University Press, 1970); sur l'immigration, voir les nombreuses références dans Robert Larin, Brève histoire du peuplement européen en Nouvelle-France (Sillery, Septentrion, 2000); D. H. Usner, "From African Captivity to American Slavery: The Introduction of Black Laborers to Colonial Louisiana ", Louisiana History, 20,1 (1979) : 25-48 et Carl A. Brasseaux et Glenn R. Conrad, dir., A Refuge for All Ages : Immigration in Louisiana History (Lafayette, Center for Louisiana Studies, University of Southwestern Louisiana, 1996); Cornelius J. Jaenen, Friend and Foe: Aspects of French-Amerindian Cultural Contact in the Sixteenth and Seventeenth Centuries (Toronto, McClelland and Stewart, 1976); Olive Patricia Dickason, Le mythe du sauvage (Sillery, Septentrion, 1993, J. Des Chênes, trad. [1984]).

11. Silvia Marzagalli, "Sur les origines de l'“Atlantic History”, paradigme interprétatif de l'histoire des espaces atlantiques à l'époque moderne», Dix-huitième siècle, 33 (2001): 17-32; Bernard Bailyn, Atlantic History: Concept and Contours (Cambridge, Harvard University Press, 2005); William O’Reilly, "Genealogies of Atlantic History», Atlantic Studies, 1,1 (2004): 66-84; Ian Steele, "Bernard Bailyn's American Atlantic", History and Theory, 46,1 (2007): 48-58.

12. De récents états des lieux, explorations ou synthèses: B. Bailyn et Patricia L. Denault, dir., Soundings in Atlantic History: Latent Structures and Intellectual Currents, 1500-1830 (Cambridge, Harvard University Press, 2009); Jack P. Greene et Philip D. Morgan, Atlantic History: A Critical Appraisal (New York, Oxford University Press, 2009); Nicholas Canny et P. D. Morgan, dir., Oxford Handbook of the Atlantic World c. 
un ensemble d'histoires, au taux de salinité variable. Mais lorsqu'elle se fait ambitieuse, cette histoire raisonne en termes d'interactions entre les habitants de tous les continents qui bornent l'océan. L'action à distance, les effets réciproques sont son gibier.

La microhistoire s'est révélée particulièrement bien adaptée à rendre de telles visées praticables, tout en illuminant des trajectoires individuelles quasi impensables auparavant ${ }^{13}$. Sous toutes ses formes, même pour mieux saisir les traces que le lointain laisse dans le local que l'on étudie, l'histoire atlantique suppose une certaine mobilité de l'observateur ainsi que de multiples points de mire ${ }^{14}$. Les historiens de la Nouvelle-France n'y échappent pas. Abordée comme partie d'un ensemble continental plus vaste dans l'histoire amérindienne, la Nouvelle-France a aussi ses connexions transocéaniques et trans-impériales. Et deux mondes - atlantique et amérindien - s'y chevauchent, au sein d'une même formation impériale ${ }^{15}$.

À peu de choses près, ces dernières années, la Nouvelle-France a servi de pôle américain à deux histoires atlantiques. D’une part, l'intégration au récit historique d'une autre population jadis marginalisée par l'historiographie a eu pour effet de situer la Nouvelle-France - et surtout la BasseLouisiane où étaient concentrés ces Africains - dans une aire transatlantique particulière: celle créée par le "passage du milieu " par où traversaient les esclaves et, en sens inverse, leurs souvenirs. Il s'agit là d'un aspect important de la redécouverte de l'histoire de la Louisiane, carrefour fascinant, depuis deux décennies ${ }^{16}$. D'autre part, l'histoire des relations entre colonies et métropole s'est renouvelée en supposant que l'Atlantique relie

1450-c. 1850 (Oxford, Oxford University Press, 2011); Douglas R. Egerton, Alison Games, Kris Lane et Donald R. Wright, The Atlantic World: A History, 1400-1888 (Wheeling, Harlan Davidson, 2007); Paul Butel, Histoire de l'Atlantique: de l'Antiquité à nos jours (Paris, Perrin, 1997).

13. Pour une microhistoire particulièrement probante, voir Jean Hébrard et Rebecca Scott, Freedom Papers: An Atlantic Odyssey in the Age of Emancipation (Cambridge, Harvard University Press, 2012).

14. Alison Games, "Atlantic History: Definitions, Challenges, and Opportunties », American Historical Review, 111,3 (2006): 741-757. Sur l’histoire connectée, voir Aline Charles et T. Wien, «Le Québec entre histoire connectée et histoire transnationale ", Globe. Revue internationale d'études québécoises, 14,2 (2011): 199-221. Sur les démarches de l'histoire atlantique: David Armitage, "Three Concepts of Atlantic History ", dans D. Armitage et Michael J. Braddick, dir., The British Atlantic World, 1500-1800 (Houndmills, Palgrave Macmillan, 2002), 11-27.

15. Catherine Desbarats, «Deux pas vers une histoire du politique décolonisée : les problèmes de la souveraineté et de l'esclavage amérindien en Nouvelle-France», Bulletin d'histoire politique, 18,3 (2010): 117-125. Sur les «formations impériales»: Ann Laura Stoler, "On Degrees of Imperial Sovereignty», Public Culture, 18,1 (2006): 125-146. Sur l'articulation des deux mondes: Paul Cohen, "Was There an Amerindian Atlantic? Reflections on the Limits of a Historiographical Concept ", History of European Ideas, 34, 4 (2008): 388-410.

16. Un échantillon assez récent: les études réunies dans Bradley G. Bond, dir., French Colonial Louisiana and the Atlantic World (Baton Rouge, Louisiana State University Press, 2005). 
autant qu'il divise. Envisager la possibilité d'une histoire transatlantique durablement partagée, par l'ancienne France et la Nouvelle, c'est bien entendu faire le pari inverse de l'histoire traditionnelle, encline à les placer sur des trajectoires historiques divergentes. Ce faisant, l'histoire commence à maîtriser son impatience de voir la métropole lâcher sa prise transatlantique, intégrant là aussi une autre temporalité ${ }^{17}$.

Consacrés à la Nouvelle-France et à l'Atlantique (ou vice-versa), les articles de ce numéro thématique illustrent à merveille l'intérêt d'une telle approche. Comme les études plus consistantes qu'ils annoncent, ces textes s'ajoutent à un nombre croissant de travaux sur la Nouvelle-France qui participent à cette réflexion collective, qu'ils revendiquent leur «atlanticité» ou non ${ }^{18}$. En guise d'entrée en matière, il n'est peut-être pas inutile d'esquisser les possibilités de la perspective atlantique. Un détour s'impose, néanmoins. Il passe par le récit traditionnel et ce regard particulier dont nous sommes tous encore un peu tributaires.

\section{D'UNE NOUVELLE-FRANCE À L'AUTRE}

Telle que formulée dans les deux Canadas, aux États-Unis et en Europe au cours d'un XIX ${ }^{\mathrm{e}}$ siècle empiétant très généreusement sur le $\mathrm{XX}^{\mathrm{e}}$, l'histoire de la Nouvelle-France a le souffle long et les variantes nombreuses. Par-delà les patriotismes et les programmes politiques contrastés, par-delà le découpage territorial des différentes histoires nationales, le regard des historiens converge sur ceux, Français de naissance ou d'origine, qui sont chargés d' "ouvrir» le territoire. Dans le cas de la Nouvelle-France, l'aventure est bien souvent présentée sous un jour improbable, ce qui fait d'ailleurs son originalité : le peu de moyens mobilisés par la métropole pour s'installer (d'aucuns disaient: se perdre) en Amérique, la vaste étendue du territoire néanmoins mis sous sa bannière ou sous sa croix, l'approche particulière des Français en matière de relations avec les Amérindiens, voilà ce qui, aux yeux des historiens, distingue cette colonisation nordaméricaine de celles des Britanniques ou des Espagnols ${ }^{19}$. Ce qui ne l'empêche pas de s'inscrire comme les autres dans le cours naturel des choses,

17. Catherine Desbarats, «La question de l’État en Nouvelle-France», dans Philippe Joutard et T. Wien, dir., Mémoires de Nouvelle-France (Rennes, Presses universitaires de Rennes, 2005), 187-198.

18. Un survol utile de parutions récentes: Christopher Hodson et Brett Rushforth, "Absolutely Atlantic: Colonialism and the Early Modern French State in Recent Historiography ", History Compass, 8,1 (2010): 101-117.

19. Allan Greer, «Première partie : historiographie», La Nouvelle-France et le monde (Montréal, Boréal, 2009, Hélène Paré, trad.), 17-76. 
dans l'accomplissement du destin des Européens puis des nouvelles nations créoles en Amérique.

Si le regard plein de convoitise que cette histoire promène sur le territoire ressemble à celui des découvreurs successifs, ce n'est pas peu souvent en raison des récits que ces derniers adressent à la cour - et à la postéritée ${ }^{20}$. Au tour des historiens, donc, de surveiller l'intérieur depuis des lieux tels que ceux, à la si royale toponymie, énumérés au début de cette introduction. Dans le récit qu'ils broderont progressivement, le commencement renferme déjà l'aboutissement: les colons ont beau le payer en épreuves de toutes sortes, le territoire qu'ils occuperont semble leur être tombé en partage dès les débuts. En attendant, bien sûr, que le retrait de la France de l'Amérique continentale ne vienne compliquer les choses.

Car comme les autres histoires coloniales des Amériques, celle-ci se préoccupe fortement de l'issue de l'aventure ${ }^{21}$. Ici nous nous éloignons des fondateurs, pour rejoindre la rupture avec la métropole, outre-Atlantique. La préoccupation avec cette coupure est d'autant plus forte, sans doute, chez les historiens de la Nouvelle-France, dont la fin - entre 1760 et 1768 selon les lieux et les événements retenus - apparaît comme déviation majeure de la trajectoire coloniale "normale» aboutissant à des républiques créoles tels les États-Unis ou les pays latino-américains. Jugé bien souvent inéluctable, ce dénouement en vient à aimanter l'histoire antérieure. C'est ainsi que jalonné de failles ou d'erreurs, ou encore de manifestations d'un génie colonial malgré tout à l'œuvre, le parcours historique de la Nouvelle-France offrira d'utiles leçons aux États-Uniens (lecteurs notamment de Parkman) ou aux Français impliqués dans de nouvelles entreprises coloniales.

À des historiographies nord-américaines faisant une assez large place aux francophones, en Louisiane, par exemple, ou au Canada anglais, ce même parcours se présentera plutôt en héritage, en prélude exotique mais indispensable à une histoire qui s'est s'accomplie depuis en atteignant sa

20. Champlain en est le plus influent exemple: Mathieu d'Avignon, Champlain et les fondateurs oubliés. Les figures du père et le mythe de la fondation (Québec, Les Presses de l’Université Laval, 2008). Peter Hulme, "Postcolonial Theory and Early America: An Approach from the Caribbean», dans Robert Blair St. George, dir., Possible Pasts: Becoming Colonial in Early America (Ithaca, Cornell University Press, 2000), 33-48.

21. Pour employer la terminologie des littéraires, si le temps raconté commence bien par des débarquements précédés de quelques préparatifs métropolitains, le temps du raconter, lui, fait d'abord passer les auteurs par la fin du Régime français. Sur la terminologie: Paul Ricœur, Temps et récit (Paris, Seuil, 1984), II : 113-120. Voir aussi Jan Eckel, «Narrativizations of the Past: The Theoretical Debate and the Example of the Weimar Republic», dans Stefan Berger et Chris Lorenz, dir., Nationalizing the Past: Historians as Nation Builders in Modern Europe (Houndmills, Palgrave Macmillan, 2010), 26-48. 
pleine dimension transcontinentale ${ }^{22}$. «L'histoire du Canada doit débuter pour ainsi dire de façon pré-natale», écrit Arthur Lower dans Colony to Nation (1946), faisant du Régime français un prologue ${ }^{23}$. Au Canada français, enfin, le récit de la Nouvelle-France fournira des gages d'accomplissements à venir, porté par le dynamisme des colons canadiens qui, au $\mathrm{XVII}^{\mathrm{e}}$ siècle déjà, ont rendez-vous avec la nation ${ }^{24}$. Voire avec l'empire, dans la mesure où leur expansionnisme s'avère rapidement plus énergique que celui de la métropole hésitante. Dans tous les cas, on interprétera la Nouvelle-France en fonction de son avenir, diversement appréhendé. La nation - choisissez laquelle - colonisera a posteriori cette histoire coloniale ${ }^{25}$.

22. Francis Parkman, The Exploration of the Great West (1869), réédité sous le titre de La Salle and the Discovery of the Great West, dans F. Parkman, France and England in North America (New York, Library of America, 1983), vol. 1, qui trouve encore des contradicteurs dans la seconde moitié du XX $\mathrm{XX}^{\mathrm{e}}$ siècle: W. J. Eccles, "The History of New France According to Francis Parkman ", dans Essays on New France (Toronto, Oxford University Press, 1987), 16-25, texte d’abord publié en 1961, et «Parkman, Francis », Dictionnaire biographique du Canada (Québec/Toronto, Les Presses de l'Université Laval/University of Toronto Press, 1990), 12: 897-902; Francis Jennings, «Francis Parkman: A Brahmin Among Untouchables», William and Mary Quarterly, 42,3 (1985): 306-328. Sur Parkman, voir notamment A. Greer, La Nouvelle-France..., op. cit., 61-76. Sur les différentes historiographies de la Nouvelle-France: Gilles Havard, «L'historiographie de la Nouvelle-France en France au cours du XX ${ }^{\mathrm{e}}$ siècle: nostalgie, oubli et renouveau ", dans T. Wien, C. Vidal et Y. Frenette, dir., De Québec à l'Amérique française. Histoire et mémoire (Québec, Les Presses de l’Université Laval, 2006), 95-124; Daniel Usner, "Between Creoles and Yankees: The Discursive Representation of Colonial Louisiana in American History ", dans B. G. Bond, French Colonial Louisiana..., op. cit., 1-21; Faye Phillips, «Writing Louisiana Colonial History in the Mid-Nineteenth Century: Charles Gayarré, Benjamin Franklin French, and the Louisiana Historical Society ", Louisiana History, 49,2 (2008): 163-190; M. Brook Taylor, Promoters, Patriots and Partisans: Historiography in Nineteenth-Century English Canada (Toronto, University of Toronto Press, 1989), 84-115, 257-259; Carl Berger, The Writing of Canadian History: Aspects of English-Canadian Historical Writing since 1900 (Toronto, University of Toronto Press, 1986), deuxième édition 17-21, 92-98, 119-126.

23. A. R. M. Lower, Colony to Nation: A History of Canada (Toronto, Longmans, Green \& Company, 1946), 1. Ce schéma faisant du Régime français une phase encore incomplète de l'histoire nationale se voit notamment dans Harold A. Innis, The Fur Trade in Canada: An Introduction to Canadian Economic History (Toronto, Univerity of Toronto Press, 1933) et sous une forme résiduelle dans Fernand Ouellet, Histoire économique et sociale du Québec 1760-1850 (Montréal, Fides, 1966), notamment page 2.

24. Le courant historiographique national insiste beaucoup sur la portée continentale du dynamisme des Canadiens (chez Groulx, les émissaires de la métropole qui font preuve d'un tel dynamisme deviennent presque des Canadiens honorifiques!). François-Xavier Garneau, Histoire du Canada depuis sa découverte jusqu'à nos jours (Québec, N. Aubin, 1845-1848), 4 vol.; Lionel Groulx, Notre grande aventure. L'empire français en Amérique du Nord (1535-1760) (Montréal, Fides, 1958); Guy Frégault, "La colonisation du Canada», dans Guy Frégault, Le XVIII siècle canadien: études (Montréal, Fides, 1968), 364-387; Guy Frégault, Iberville le conquérant (Montréal, Éditions Pascal, 1944); Guy Frégault, La guerre de la Conquête 1754-1760 (Montréal, Fides, 1955); sur l’embryon national: Jean Blain, «Économie et société en NouvelleFrance : l'historiographie des années 1950-1960. Guy Frégault et l'école de Montréal ", RHAF, 28,2 (1974): 178-183; le Canadien de W. J. Eccles est un proche parent de celui de Frégault. Voir par exemple W. J. Eccles, The Ordeal of New France: 13 Radio Scripts (Montréal, CBC International Services, 1966).

25. Sur cette "vision rétrospective», voir Frederick Cooper, Le colonialisme en question. Théorie, connaissance, histoire (Paris, Payot, 2010), 29-30; Joyce Chaplin, «Expansion and Exceptionalism... », op. cit. 
Aussi l'ancrera-t-elle en Amérique, en marquant le tracé des frontières spatiales. Ici nous pensons moins à la tendance des différentes histoires nationales à confondre avec la Nouvelle-France le territoire de la patrie qu'elles cherchent à inscrire dans le temps long ${ }^{26}$. Nous pensons davantage à une façon de situer la Nouvelle-France entre Atlantique et continent en fonction de l'avenir qui l'attend au détour. À l'est, elle semble déjà s'éloigner de la France, Conquête/ cession appréhendée oblige, dans une dérive qui a quelque chose de tectonique ${ }^{27}$. Pendant que cette "frontière» s'affirme avec le temps, une autre semble s'effacer. Les lignes séparant les zones francisées du Canada ou de la Basse-Louisiane de l'intérieur du continent, terre promise à la France en quelque sorte, ne sont que provisoires. Au-delà, les Français et leurs descendants créoles sont certes peu nombreux, mais ne sont-ils pas déjà chez eux? De la sorte et malgré le caractère hésitant du peuplement français, toute l'entreprise coloniale semble vouée à se déplacer vers le centre du continent. De façon implicite ou explicite, elle épouse la marche vers l'avenir des créoles, associant au passé les métropolitains laissés derrière. Les Amérindiens, eux, sont laissés devant pour l'instant, mais leur écartement n'en est pas moins programmé ${ }^{28}$.

Comme les histoires des autres Amériques coloniales, ce récit s'est effiloché avec le temps, reflétant en cela la marche du monde depuis un bon demi-siècle. Décolonisation puis mondialisation à l'extérieur, mouvements contre diverses formes d'exclusion à l'intérieur, les deux convergeant lors des débats sur la redéfinition de la nation ${ }^{29}$, tout cela a interpellé les historiens - et bien entendu, pas que ceux de la Nouvelle-France. Le regard historien s'est diversifié. L'histoire de cette formation coloniale s'en

26. A. Greer, «National, Transnational, and Hypernational Historiographies: New France Meets Early American History", Canadian Historical Review, 91,4 (2010): 695-724; A. Greer, La Nouvelle-France..., op. cit., 17-46.

27. C'est le cas même des historiens qui voient l'histoire coloniale comme un prolongement de celle de la France. Marcel Giraud, par exemple, toujours soucieux d'expliquer pourquoi la colonisation de la Louisiane est si hésitante (se soldant donc par la cession), ne perd jamais de vue la rupture ultime entre la France et cette partie de son premier empire. M. Giraud, Histoire de la Louisiane française (Paris, PUF, 1953-1974), 4 vol. et M. Giraud, A History of French Louisiana, vol. 5, Brian Pearce, trad. (Baton Rouge, Louisiana State University Press, 1987). Stimulant sur les vases communicants espace/temps, même si sa comparaison entre les historiographies allemande et québécoise rapproche à notre avis trop les deux cas: Chris Lorenz, «Double Trouble: A Comparison of the Politics of National History in Germany and in Québec », dans S. Berger et C. Lorenz, dir., Nationalizing..., op. cit., 49-70.

28. Voir Johannes Fabian, Time and the Other: How Anthropology Makes Its Object (New York, Columbia University Press, 2002 [1983]).

29. Gérard Bouchard, Genèse des nations et cultures du Nouveau Monde: essai d'histoire comparée (Montréal, Boréal, 2000); Craig Calhoun, Nations Matter: Culture, History, and the Cosmopolitan Dream (Londres/New York, Routledge, 2007). 
trouve plus pluraliste et moins tributaire de la nation créole - ou métropolitaine - si impatiente de naître. La Nouvelle-France qui émerge actuellement est plus compliquée que l'ancienne et vraisemblablement plus instable. Et pourtant, sa fin semble moins inéluctable, comme si l'attention prêtée à tous ces mouvements latéraux avait ralenti le cours de l'histoire, permettant d'imaginer d'autres avenirs. Et d'échapper au moins partiellement à «l'illusion rétrospective de fatalité» qui marquait autrefois l'historiographie ${ }^{30}$.

Un aspect du changement: dans sa façon de situer la Nouvelle-France entre Atlantique et continent, l'historiographie plus récente a tendance à inverser le schéma frontalier que nous venons d'esquisser. Tout se passe comme s'il y a eu échange de cloisons: devenue moins perméable à la présence française, l'Amérique amérindienne (pourtant présente jusqu'en pleine zone francisée) est séparée de celle-ci par la frontière fondamentale que représentent leurs relations respectives avec le colonialisme métropolitain. Face à cette mer de l'Ouest amérindienne, quelque chose ressemblant à un mare nostrum atlantique, bornée par la France et ses colonies américaines, prend forme dans les études historiques. Un rapprochement transatlantique analogue s'est opéré dans les latitudes afro-louisianaises. Ces dernières années, les études adoptant la perspective atlantique se sont multipliées, abordant les communications, le commerce, la religion ou l'État, pour ne citer que ces exemples. Et l'Histoire de l'Amérique française de Gilles Havard et Cécile Vidal constitue une première synthèse réussie, à mi-chemin entre Atlantique et continent ${ }^{31}$.

C'est justement l'Atlantique (Nord, en l'occurrence) que les articles de ce numéro traversent à répétition. Que signifie ce changement de perspective? De manière impressionniste, et privilégiant les migrations et

30. Citation: Raymond Aron, Philosophie critique de l'histoire (Paris, Vrin, 1969), 186-187, quatrième édition, cité dans Paul Ricœur, Temps et récit (Paris, Seuil, 1983), I: 263. Mouvements latéraux: Bernard Bailyn, «Introduction: Reflections on Some Major Themes", dans B. Bailyn et Patricia L. Denault, dir., Soundings in Atlantic History: Latent Structures and Intellectual Currents, 1500-1830 (Cambridge, Harvard University Press, 2009), 2 ; Renate Pieper et Peer Schmidt, «Introduction », dans R. Pieper et P. Schmidt., dir. Latin America and the Atlantic World (Cologne, Böhlau, 2005), 16-17.

31. Kenneth J. Banks, Chasing Empire Across the Sea: Communications and the State in the French Atlantic, 1713-1763 (Montréal/Kingston, McGill-Queen’s University Press, 2002); Catherine Ferland, Bacchus en Canada. Boissons, buveurs et ivresses en Nouvelle-France, (Québec, Septentrion, 2010); Dominique Deslandres, Croire et faire croire. Les missions françaises au XVII siècle (1600-1650) (Paris, Fayard, 2003); Allan Greer, Catherine Tekakwitha et les jésuites. La rencontre de deux mondes (Montréal, Boréal, 2007), traduction de Hélène Paré; Louise Dechêne, Le partage des subsistances au Canada sous les Régime français (Montréal, Boréal, 2004); Louise Dechêne, Le peuple, l'État et la guerre au Canada sous le Régime français (Montréal, Boréal, 2008); Shannon Lee Dawdy, Building the Devil's Empire: French Colonial New Orleans (Chicago, University of Chicago Press, 2008); G. Havard et C. Vidal, op. cit. 
l'exercice du pouvoir royal, cherchons à voir comment les perspectives offertes par l'approche atlantique modifient ou pourraient modifier l'horizon d'attente de l'historiographie. À quoi l'approche atlantique peut-elle servir?

\section{DES ATLANTIQUES DE LA NOUVELLE-FRANCE}

Un premier exemple de ce qu'apporte la prise en compte de la dimension atlantique de l'histoire de la Nouvelle-France se voit au large de la Louisiane et, plus précisément, de la Basse-Louisiane où était concentrée la population d'origine africaine ${ }^{32}$. Très majoritairement en esclavage sous le Régime français et objectivé par l'histoire traditionnelle, ce groupe a beaucoup attiré l'attention des historiens au cours des deux dernières décennies, alors que l'histoire louisianaise vivait une sorte de renaissance. Il a également joué un certain rôle dans la discussion plus vaste sur l'expérience transatlantique des esclaves, motif de plus de s'intéresser à lui ici. Pendant longtemps, l'horizon africain n'attirait guère le regard des historiens de la Louisiane. Il faut dire que la majeure partie de ceux, de plus en plus nombreux à partir des années 1970, qui s'intéressaient à l'histoire des esclaves en Amérique portaient un intérêt plutôt superficiel à l'Afrique; à partir des années 1970, la thèse de la créolisation voyait dans le brassage des populations opéré par la traite un obstacle majeur à la conservation d'éléments importants des cultures d'origine ${ }^{33}$. La remise en question de cette interprétation prit de l'ampleur vers 1990, grâce notamment à l'intérêt accru des historiens de l'Afrique envers l'expérience de la diaspora originaire de ce continent ${ }^{34}$. La possibilité de voir ressurgir au moins des "principes culturels» africains là où des esclaves d'une même origine ethnique ou régionale se retrouvaient en Amérique allait aussi

32. Sur les Africains ailleurs en Nouvelle-France: M. Trudel, L'esclavage..., op. cit.; Denise BeaugrandChampagne, Le procès de Marie-Josèphe-Angélique (Outremont, Libre Expression, 2004); Cécile Vidal, "Africains et Européens au Pays des Illinois durant la période française (1699-1765)», French Colonial History, 3 (2003): 51-68; et de façon plus tangentielle dans Brett Rushforth, Bonds of Alliance: Indigenous and Atlantic Slaveries in New France (Chapel Hill, University of North Carolina Press, 2012).

33. Sidney Mintz et Richard Price, An Anthropological Approach to the Afro-American Past: A Caribbean Perspective (Philadephie, Institute for the Study of Human Issues, 1976); précurseur de l'approche transatlantique: Walter Rodney, «Upper Guinea and the Significance of the Origins of Africans Enslaved in the New World", Journal of Negro History, 54,4 (1969): 327-345.

34. Voir notamment John Thornton, Africa and Africans in the Making of the Atlantic World, 1400-1800 (New York, Cambridge University Press, 1998 [1992]), deuxième édition. Sur l'historiographie africaine, Joseph C. Miller, «History and Africa/ Africa and History », American Historical Review, 104,1 (1999): 1-32; Kristin Mann, "Shifting Paradigms in the Study of the African Diaspora and of Atlantic History and Culture», Slavery \& Abolition, 22,1 (2001): 3-21. 
mettre l'étude de tels regroupements au programme de ceux qui montaient de volumineuses bases de données sur le commerce des esclaves ${ }^{35}$.

Mais des historiens des colonies s'y intéressaient également, et c'est ainsi que la Louisiane, colonie pourtant située en retrait des principaux circuits du commerce des esclaves, allait faire parler d'elle ${ }^{36}$. Paru en 1992, l'influent livre Africans in Colonial Louisiana de Gwendolyn Midlo Hall plaidait pour la conservation en Louisiane de traits culturels africains spécifiques $^{37}$. Cette interprétation venait appuyer une caractérisation plus globale de l'esclavagisme louisianais. D’après Hall, l'anémie du secteur des plantations sous le Régime français et le désordre ambiant favorisaient une fluidité inégalée des relations sociales et des attitudes raciales dans la colonie. Ces conditions ainsi que l'arrivée en peu d'années d'une masse critique d'esclaves embarqués sur la côte sénégambienne étaient propices à la conservation de certains éléments du patrimoine linguistique, musical ou religieux, principalement de cette région d'Afrique. À la longue, la Louisiane serait même devenue le plus important foyer d'influences africaines de toutes les colonies nord-américaines.

Les travaux publiés depuis - ou en même temps, comme le remarquable Indians, Settlers, and Slaves in a Frontier Exchange Economy de Daniel Usner n'offrent pas de prise globale sur la conservation d'éléments culturels

35. David Eltis et David Richardson, dir., Routes to Slavery: Direction, Ethnicity, and Mortality in the Transatlantic Slave Trade (Londres, Frank Cass, 1997); D. Eltis et D. Richardson, Atlas of the Transatlantic Slave Trade (New Haven, Yale University Press, 2010); Gwendolyn Midlo Hall, Slavery and African Ethnicities in the Americas : Restoring the Links (Chapel Hill, University of North Carolina Press, 2005). La principale base de données se trouve à l'adresse www.slavevoyages.org.

36. Entre 1700 et 1760 - et à peu de choses près, entre 1720 et 1731 - la Louisiane aurait reçu moins de un pour cent des Africains qui traversèrent l'Atlantique vers les Amériques au cours des six décennies. Les quelque 6000 personnes concernées survécurent et firent des enfants en nombre suffisant pour devenir et rester majoritaires parmi les habitants non amérindiens de la colonie. Thomas N. Ingersoll, «The Slave Trade and the Ethnic Diversity of Louisiana's Slave Community », Louisiana History, 37,2 (1996): 133-161; David Eltis, "The Volume and Structure of the Transatlantic Slave Trade : A Reassessment », William and Mary Quarterly, 58,1 (2001): 17-46; James Pritchard, D. Eltis et D. Richardson, "The Significance of the French Slave Trade to the Evolution of the French Atlantic World before 1716», dans D. Eltis et D. Richardson, dir., Extending the Frontiers : Essays on the Transatlantic Slave Trade Database (New Haven, Yale University Press, 2008), 205-227; Paul LaChance, "The Growth of the Free and Slave Populations of French Colonial Louisiana ", dans B. G. Bond, French Colonial Louisiana, op. cit., 204-243.

37. Gwendolyn Midlo Hall, Africans in Colonial Louisiana: The Development of Afro-Creole Culture in the Eighteenth Century (Baton Rouge, Louisiana State University Press, 1992). Outre de déclencher un débat sur la nature de l'esclavagisme louisianais, cette démonstration pionnière fournit un exemple régional éloquent à deux importantes synthèses sur les esclaves nord-américains. Ira Berlin, Many Thousands Gone: The First Two Centuries of Slavery in North America (Cambridge, Harvard University Press, 1998); Michael A. Gomez, Exchanging Our Country Marks: The Transformation of African Identities in the Colonial and Antebellum South (Chapel Hill, University of North Carolina Press, 1998). 
africains en Louisiane ${ }^{38}$. Ils insistent tantôt sur la dureté de l'esclavagisme louisianais et sur l'émergence du racisme, tantôt sur l'existence de sphères de liberté relative pour au moins certains esclaves, sans parler d'un nombre croissant de gens libres de couleur ${ }^{39}$. L'ensemble du dossier pointe sans doute davantage vers la transformation en Louisiane des éléments africains spécifiques, en raison notamment de l'origine ethnique contrastée de nombre de couples d'esclaves ${ }^{40}$.

C’est plutôt le statut de vecteurs culturels privilégiés que Hall attribuait aux membres d'un groupe ethnique particulier qui a provoqué une réflexion plus poussée sur l'expérience migratoire des esclaves échoués en Louisiane. Ces gens, les Bambaras, appartenaient en principe à la culture mandé de la "grande courbe» du Niger, loin de la côte sénégambienne. Réputés belliqueux sur un continent puis insoumis sur l'autre, ils étaient plutôt visibles dans les sources louisianaises. Dans la foulée de la parution de l'étude de Hall, on ne tarda pas à souligner que l'ethnonyme «Bambara» manquait de précision: en Afrique, il pouvait inclure bon nombre d'autres esclaves non musulmans, sans pour autant former la majorité, plutôt d'origine côtière pendant ces années, des esclaves expédiés en Louisiane depuis la Sénégambie. Si les Bambaras semblaient jouer un rôle important en Louisiane, c'est aussi qu’après leur arrivée dans la colonie, certains Africains d'origine différente s'étaient ralliés à eux ${ }^{41}$.

38. D. H. Usner, Indians..., op. cit.; voir aussi du même auteur, «Indian-Black Relations in Colonial and Antebellum Louisiana ", dans Stephan Palmié, dir., Slave Cultures and the Cultures of Slavery (Knoxville, University of Tennessee Press, 1995), 145-161.

39. Dépeignant (pace Hall) un esclavagisme néo-orléanais qui ne cède rien en dureté à ceux des colonies anglo-américaines: Thomas N. Ingersoll, Mammon and Manon in Early New Orleans: The First Slave Society in the Deep South, 1718-1819 (Knoxville, University of Tennessee Press, 1999); T. N. Ingersoll, "The Slave Trade... », op. cit. Sur la brutalité : Cécile Vidal, «De l'incorporation à l'exclusion. Les relations entre Amérindiens, Européens et Anglo-Américains dans la vallée du Mississippi de 1699 à 1830 ", La Revue Tocqueville, 25,2 (2004): 35-54; S. L. Dawdy, Building..., op. cit., 178-181. Racisme: Guillaume Aubert, " "The Blood of France": Race and Purity of Blood in the French Atlantic World", William and Mary Quarterly, 61,3 (2004) : 439-478; Cécile Vidal, «Francité et situation coloniale: Nation, empire et race en Louisiane française (1699-1769)", Annales HSS, 63,5 (2009): 1019-1050; Cécile Vidal, "Private and State Violence Against African Slaves in Lower Louisiana During the French Period, 1699-1769", dans Thomas J. Humphrey et John Smolenski, dir., New World Orders: Violence, Sanction, and Authority in the Colonial Americas (Philadelphie, University of Pennsylvania Press, 2005), 92-110 et 306-310; S. L. Dawdy, Building..., op. cit, 181-185. Sphères de liberté: Emily Clark et Virginia Meacham Gould, "The Feminine Face of Afro-Catholicism in New Orleans, 1727-1852", William and Mary Quarterly, 59,2 (2002): 409-448; E. Clark, Masterless Mistresses: The New Orleans Ursulines and the Development of a New World Society, 1727-1834 (Chapel Hill, University of North Carolina Press, 2007). Un bilan: G. Havard et C. Vidal, Histoire..., op. cit., 475-534.

40. G. Havard et C. Vidal, Histoire..., op. cit., 493-496.

41. Stephan Palmié, "Compte rendu de Hall, Africans in Colonial Louisiana», Africa, 64 (1994): 168-171; Peter Caron, " "Of a Nation Which the Others Do Not Understand": Bambara Slaves and African Ethnicity in Colonial Louisiana, 1718-60», Slavery \& Abolition, 18,1 (1997): 115. 
Kevin Roberts concluait de ces observations que l'ethnie bambara était devenue un pôle d'attraction auprès des esclaves non musulmans en Louisiane ${ }^{42}$.

C’est en pointant des éléments culturels précis qui auraient survécu à l'expérience du "passage du milieu», que Hall avait jeté un pont entre l'Afrique et la Louisiane ${ }^{43}$. La critique, elle, avait plutôt signalé la continuité transatlantique d'un processus de formation et de transformation des ethnies, de brouillage et de recomposition des traits. Il n'est pas sûr que la réputation de l'esclavagisme louisianais ait gagné au change : l'importance que les migrants involontaires accordaient à l'appartenance ethnique - quitte à s'en faire imposer une en chemin - ressemble davantage à une stratégie de survie sous l'esclavagisme, où qu'il se produise. C'est du moins la conclusion que tirent des interprétations plus générales de ce processus $^{44}$. Quoi qu'il en soit, la conclusion voulant que c'est l'ethnicité qui traverse l'Atlantique et non des ethnies figées nous oblige à réviser nos attentes en nous rapprochant de l'histoire personnelle des esclaves ${ }^{45}$. Et par là, à nous rapprocher de l'Afrique, aussi. Non pas en vecteurs mais en acteurs s'appuyant sur leur expérience africaine, ces émigrants malgré eux essaient de rendre moins dangereusement nouveau le monde américain.

Les travaux portant sur une autre sphère atlantique, celle qui englobe la France et la Nouvelle-France, amènent à faire des rapprochements analogues. Deux exemples démontrent l'intérêt d'une démarche qui s'attarde, davantage que ne le faisait l'histoire traditionnelle, aux connexions entre les deux rives de cet espace océanique: les migrations et l'exercice du pouvoir royal.

C'est dans l'optique du peuplement, produisant des colons prêts à se mesurer à l'Amérique et y laisser une descendance, que l'histoire a longtemps abordé les migrations transatlantiques des Européens. L’inévitable

42. Kevin Roberts, "Africa in Louisiana: In Search of "Bambara" and Creole Identities in Literary and Statisical Sources ", dans Toyin Falola et Christian Jennings, dir., Sources and Methods in African History: Spoken, Written, Unearthed (Rochester, University of Rochester Press, 2003), 156-165.

43. Hall s'attaque à ces problèmes à une échelle plus vaste dans Slavery..., op. cit. Voir aussi G. M. Hall, "African Ethnicities and the Meaning of "Mina" ", dans Paul E. Lovejoy et David V. Trotman, dir., Transatlantic Dimensions of Ethnicity in the African Diaspora (Londres/New York, Continuum, 2003), 65-81.

44. Paul E. Lovejoy, «Ethnic Designations of the Slave Trade and the Reconstruction of the History of Transatlantic Slavery", dans Paul E. Lovejoy et D. V. Trotman, dir., Transatlantic Dimensions..., op. cit., 9-42; James Sidbury et Jorge Cañizares-Esguerra, «Mapping Ethnogenesis in the Early Modern Atlantic», William and Mary Quarterly, 68,2 (2011): 181-208.

45. Voir la belle étude microhistorique de Sophie White, "Wearing three or four handkerchiefs around his collar, and elsewhere about him": Slaves' Constructions of Masculinity and Ethnicity in French Colonial New Orleans ", Gender \& History, 15,3 (2003): 528-549. 
comparaison avec les Treize Colonies et la retentissante tragédie louisianaise associée au nom de John Law plaçaient le peuplement de la NouvelleFrance sous le signe de la difficultét ${ }^{46}$. L'ambitieux projet d'étude de la population de la vallée laurentienne, lancé vers 1970 par les démographes montréalais, privilégiait lui aussi les immigrants qui avaient «fait souche » - et des enfants ${ }^{47}$.

C’est plus récemment que le domaine d'étude s'est élargi pour inclure d'autres migrants et mieux intégrer le pays d'origine - en partie grâce aux données individuelles sur les fondateurs de lignées canadiennes rassemblées par les Montréalais. Du coup, l'axe de peuplement est devenu un véritable champ migratoire. C'est donc encore une fois le Canada qui a bénéficié d'une attention toute particulière de la part des spécialistes. En 1983, le démographe Mario Boleda exploita la banque montréalaise pour démontrer que la majorité des Français - à forte prépondérance masculine, en effet - qui atteignirent la vallée laurentienne repartirent après quelques saisons $^{48}$. Le nombre réduit - du moins dans l'optique impériale - de ceux qui s'installèrent à demeure au Canada reflétait alors non seulement la faible propension des Français à traverser l'Atlantique vers la vallée laurentienne, mais aussi leur forte propension à le retraverser. Dans la foulée, Peter Moogk et surtout Leslie Choquette se penchèrent sur le contexte de départ de ces migrations. Ces études présentaient la traversée de nombre de jeunes gens vers le Canada ou l'Acadie comme prolongement des courants de migration de main-d'œuvre internes à la France. Si tel était le cas, une proportion importante de ces hommes n'était pas des émigrants vite déçus par le gel précoce ou la pénurie de Canadiennes prêtes à les épouser, mais des travailleurs migrants explorant les marges extrêmes du marché français de l'emploi temporaire ${ }^{49}$. L'élargissement

46. Bien que l'historiographie s'intéressant aux Amérindiens voie là un des facteurs favorisant des relations plus équilibrées qu'ailleurs en Amériques entre Français et autochtones.

47. Hubert Charbonneau et al., Naissance d'une population: les Français établis au Canada au XVII siècle (Paris/Montréal, INED/Presses de l’Université de Montréal, 1987).

48. Mario Boleda, «Les migrations au Canada sous le régime français », thèse de Ph.D. en démographie, Université de Montréal, 1983; Mario Boleda, "Trente mille Français à la conquête du Saint-Laurent», Histoire sociale/Social History, 23,45 (1990): 153-177; Leslie P. Choquette, «French Emigration to Canada in the $17^{\text {th }}$ and $18^{\text {th }}$ Centuries ", thèse de Ph.D. en histoire, Université Harvard, 1988; Peter N. Moogk, «Reluctant Exiles: Emigrants from France in Canada before 1760 », William and Mary Quarterly, 46,3 (1989) : 463-505; Peter N. Moogk, «Manon’s Fellow Exiles: Emigration from France to North America Before 1763 ", dans Nicholas P. Canny, dir., Europeans on the Move: Studies on European Migration, 1500-1800 (Oxford, Clarendon Press, 1994), 236-260. Chiffres révisés dans Yves Landry, «Les Français passés au Canada avant 1760 : le regard de l'émigrant ", Revue d'histoire de l'Amérique française, 59,4 (2006) : 482-484.

49. Leslie Choquette, De Français à paysans. Modernité et tradition dans le peuplement du Canada français (Sillery/Paris, Septentrion/Presses de l'Université de Paris-Sorbonne, 2001 [1997]), trad. Gervais Carpin. 
de l'échelle d'analyse pour englober les deux côtés de l'Atlantique permet donc, non sans paradoxe, de mieux comprendre des décisions individuelles qu'une historiographie impatiente devant les lenteurs du peuplement français avait explicitement ou implicitement déplorées. Elle incite à rapprocher ces engagés d'autres travailleurs qui n’hésitaient pas à traverser et à retraverser l'Atlantique, pour exploiter la pêche terre-neuvienne, par exemple - et d'ajuster nos attentes en conséquence ${ }^{50}$.

Le pouvoir royal et plus largement institutionnel est un autre phénomène qui se situe avantageusement dans le cadre d'une histoire atlantique. Penser l'Ancien Régime français comme entité supra-atlantique permet de visualiser la Nouvelle-France comme partie intégrante d'une formation impériale plus vaste. Non pas pour nécessairement nier l’influence de la distance transatlantique, de la relative lenteur des communications, voire du statut colonial, mais pour ne pas préjuger de leur influence et donc, chercher à identifier les circonstances dans lesquelles ils jouent ${ }^{51}$. Ou, pour inverser le sens géographique de la réflexion, procéder ainsi permet d'incorporer les colonies nord-américaines à la zone où se construit l'État moderne $^{52}$. Si l'on s'y permet certes des expériences particulières, la Nouvelle-France prolonge, malgré tout, la France où l'Ancien Régime travaillait déjà sur lui- même ${ }^{53}$.

L'ouvrage pionnier de Kenneth Banks nous a justement permis d'imaginer un tel espace atlantique français étatique. En scandant le rythme et l'intensité du dialogue épistolaire entre agents royaux métropolitains et leurs homologues ou inférieurs basés à Québec, la Nouvelle-Orléans, ou Saint-Pierre en Martinique, Chasing Empire Across the Sea explorait certaines pratiques constitutives de cette formation impériale ${ }^{54}$. Au-delà des circuits administratifs, les travaux de Brett Rushforth sur l'esclavage amérindien en Nouvelle-France révèlent d'autres aspects de la nature du pouvoir exercé à une échelle transatlantique. Deux traits sautent aux yeux

50. C'est en partie l'optique populationniste qui encourage les historiens à constater l'échec de l'engagement transatlantique pour le Canada, alors qu'en tant que système de recrutement il a vraisemblablement réussi à s'ajuster à peu près à la demande coloniale. C'est du moins ce qui ressort des observations de P. Moogk et de celles d'Arnaud Bessière, «La domesticité dans la colonie laurentienne au XVII ${ }^{\mathrm{e}}$ siècle et au début du XVIII ${ }^{\mathrm{e}}$ siècle (1640-1710)", thèse de Ph.D. en histoire, Université du Québec à Montréal et Paris-Sorbonne, 2007.

51. Jean-Frédéric Schaub, "La catégorie "études coloniales" est-elle indispensable?», Annales HSS, 63,3 (2008): 625-646.

52. C. Desbarats, «La question...», op. cit.

53. Denis Richet, «La monarchie au travail sur elle-même», De la Réforme à la Révolution. Études sur la France moderne (Paris, Aubier, 1991), 425-445.

54. Kenneth Banks, op. cit. 
tout au long de son ouvrage fascinant: d'une part la pluralité des espaces légaux, mais d'autre part, leur profonde interdépendance, d'où l'intérêt de les explorer sous une même loupe ${ }^{55}$.

La confirmation, en 1709 dans un acte passé à Québec (et qui doit «être lu à Montréal et à Trois Rivières comme à Québec») du droit de posséder en "plaine propriété" "Panis» et "Negres» dans "cette colonie» incarne à elle seule tout un pan de l'impérialisme français, et illustre les propos de Rushforth concernant le pluralisme légal: la loi en question est un chef-d'œuvre de bricolage judiciaire, et de manipulation opportuniste de catégories juridiques, identitaires et même géographiques au service de "l'utilité» (c'est le langage du document...) économique. Son auteur, l'intendant Jacques Raudot, invoque le principe que «toutes les colonies doivent être sur le même pied», alors que le statut d'esclaves amérindiens est loin d'être clair à la même époque dans les Antilles françaises, ou plus tard, en Louisiane. Pour composer avec cette dernière incohérence, le texte improvise une catégorie particulière, mais floue, d'Amérindiens lointains et ennemis, «les Panis», distincts, donc, des nations alliées qu’on ne peut soumettre légalement à l'esclavage. On répète ainsi un discours mis à l'épreuve en Afrique.

Et le parallèle justificatif se poursuit explicitement: comme les «Negres» (habitants de la Nigrite continentale imaginée par les Européens, loin des forts et comptoirs qu'ils fréquentent), les "Panis» seront d'une grande utilité, permettant la floraison des entreprises coloniales menacées par un manque de main-d'œuvre. Et pour rapprocher de plus près ces deux sortes d'humains qu'on est en droit de posséder, l'ordonnance défend du même coup aux "esclaves Panis et Negres» de "quitter leurs maitres soub pretexte qu'il n'y a pas d'esclaves en France». Quoique ce dernier principe, tergiverse paresseusement l'ordonnance, ne soit "pas toujours vray $^{56}$ ». Notons enfin ceci: alors que le baptême forge parfois la parenté fictive au cour des alliances franco-amérindiennes de l'Amérique du Nord, nulle mention ici du catholicisme pourtant si présent dans la rhétorique justificative de cette pièce maîtresse de la régulation judiciaire de l'esclavage aux Antilles françaises, qu'était le "code noir» de 1685. Au gré d'ellipses et de contradictions, l'intendant de la Nouvelle-France exploite ainsi les ambigüités légales et sociales de l'espace atlantique français, tout en les amplifiant. Impossible, en d'autres termes, de comprendre l'escla-

55. B. Rushforth, Bonds of Alliance..., op. cit.

56. Jacques Raudot, "Ordonnance concernant l'esclavage au Canada», 13 avril 1709. Bibliothèque et Archives nationales du Québec, Centre de Québec, Ordonnances des intendants, E1, S1, P509. 
vage amérindien au Canada sans faire de détours en France, en Afrique et aux Antilles.

Nous sommes d'autant plus ici dans un espace où les ambigüités qui naissent ou renaissent dans le domaine américain de Sa Majesté reflètent, mais éclairent aussi l'évolution de l'exercice du pouvoir au sein du royaume même. En sens inverse, une meilleure prise en considération des pratiques de la "monarchie intégratrice» en France permet de mieux apprécier l'originalité des défis que la Nouvelle-France créole et amérindienne lance à l'absolutisme. Par exemple, l'accent que nombre d'études récentes mettent sur les négociations de la monarchie absolue avec des pouvoirs intermédiaires métropolitains constitue une toile de fond indispensable à l'émergence en colonies de constellations d'intérêts diverses, plus ou moins de mèche avec les représentants locaux du roi ${ }^{57}$.

De façon analogue, il n'est pas sûr si la souveraineté amérindienne, thème central de beaucoup d'études récentes sur le centre du continent, doit toujours être interprétée comme la mise en échec de l'omniprésente volonté d'hégémonie royale; dans certains cas du moins, elle serait plutôt acceptée par une monarchie habituée à composer avec des degrés d'hégémonie très variés, surtout là où elle estime que les puissances européennes rivales ne seraient pas avantagées ${ }^{58}$.

Bref, le regard vers la France permet de modifier nos attentes envers l'absolutisme tel qu'il s'exerce - ou ne s'exerce guère - en Nouvelle-France. Et cette dernière, combinaison de territoires mal délimités où le roi règne tant bien que mal ou exerce, voire prétend exercer de l'influence, paraît prolonger, certes en plus contrastée, la complexité légale métropolitaine.

Les cinq textes réunis ici rejoignent de telles tentatives d'envisager la Nouvelle-France comme faisant partie d'une entité supra-atlantique, politique, légale, certainement, mais aussi religieuse. Et à force de vouloir revisiter, ou transcender les frontières historiographiques traditionnelles de la Nouvelle-France, ils en viennent parfois à réexaminer, littéralement, la question de frontières telles qu'elles sont vécues et conçues à l'époque moderne.

D’entrée de jeu, Catherine Desbarats et Allan Greer posent une question simple, et géographique: "Où est la Nouvelle-France»? La réponse

57. Sur la France, voir le survol de William Beik, «The Absolutism of Louis XIV as Social Collaboration", Past \& Present, 188 (2005): 195-224; sur les groupes d'intérêts coloniaux, notamment les lectures de la politique louisianaise de K. Banks, Chasing Empire..., op. cit., et de S. L. Dawdy, Building..., op. cit.

58. Ces remarques s'inspirent de l'analyse de L. Benton, A Search for Sovereignty..., op. cit., dont il sera plus longuement question plus loin. 
ne pourra être aussi lapidaire que l'interrogation de départ; les lecteurs sont invités à explorer pourquoi, depuis les premières apparitions du toponyme dans la cartographie européenne des Amériques, jusqu’à son absence frappante du traité de Paris en 1763. Tout au long, les auteurs documentent l'irréductible ambigüité territoriale de la Nouvelle-France, et offrent des éléments d'interprétation. Ils s'inspirent, entre autres, de fertiles réflexions transhistoriques sur la nature des empires, qui comme les «États» auxquels ils sont associés, partagent le trait d'être en devenir constant. Et de telles "formations impériales», insiste vigoureusement l'anthropologue Ann Stoler, "ne sont pas maintenant, et ont rarement été, des régimes politiques (ou "polities" dans le sens que l'entend la philosophie politique) aux bornes ou frontières évidentes ${ }^{59 "}$; au contraire, la superposition de souverainetés qu'impliquent les formations impériales exige, et génère, de telles ambigüités. Faut-il s'étonner que Timothy Pearson trouve, dans le dernier texte de ce numéro, une opacité parallèle dans l'organisation des missions religieuses séculières?

Les formations impériales ont néanmoins leur géographie. Avec les ethnohistoriens, qui s'acharnent à recouvrir les espaces politiques et sociaux des Amérindiens, les historiens du droit sont parmi ceux qui ont le plus vigoureusement renouvelé l'histoire spatiale des empires de l'époque moderne ${ }^{60}$. Le droit, ou mieux, le déploiement d'arguments et d'instruments légaux, note Lauren Benton, jouent un rôle primordial dans les tentatives de fabriquer la souveraineté impériale à travers les Amériques (et bien au-delà), et en déterminent du moins partiellement l'empreinte sur la terre et sur l'eau. Les nouvelles chartes de compagnies constituent et attribuent des droits de circuler et de commercer dans des corridors maritimes et fluviaux; ils confèrent en même temps des droits de gouverner, de construire des places fortes dans des sites ponctuels, stratégiques, avec accès facile à ces corridors; les contrats de pêche transatlantique, sanctionnés par des amirautés européennes, font aussi voyager le droit, tandis que les sanctions royales accordées aux missionnaires, et les commissions octroyées aux capitaines de navires, tracent à leur tour des zones privilégiées de contrôle impérial. La géographie résultante est

59. Dans l'anglais original: «[...] imperial formations are not now, and rarely have been clearly bounded or bordered polities». A. L. Stoler, «On Degrees of Imperial Sovereignty», loc. cit., 128.

60. Pour les Grands Lacs, Heidi Bohaker, «Nindoodemag: The Significance of Algonquian Kinship Networks in the Eastern Great Lakes' Region, 1600-1701 ", William and Mary Quarterly, 63 (2006): 23-52. Rushforth tente aussi de reconstituer, à travers l'analyse des pratiques algonquiennes de captivité, le tracé de frontières sociales dans son Bonds of Alliance..., op. cit. Voir aussi Michael Witgen, An Infinity of Nations. How the Native New World Shaped Early America (Philadelphie, University of Pennsylvania Press, 2011). 
hétéroclite, truffée de discontinuités territoriales, un amalgame de corridors et d'enclaves, tels les forts, les comptoirs ou les missions ${ }^{61}$. Nous sommes loin, pourrait-on ajouter avec Benton, d'un contrôle "rationnel», systématiquement réparti à travers l'espace. Nous sommes beaucoup plus proches, en fait, des monarchies «composites» de l'Europe moderne, qui loin d'être constituées de territoires bornées de façon simple et linéaire, cumulent des juridictions et des possessions hétérogènes (seigneuries, etc. ${ }^{62}$. Il est significatif, par ailleurs, qu'encore au milieu du XVIII ${ }^{e}$ siècle et même au-delà, le terme "frontière» en France conserve sa signification première de lieux mouvants d'affrontement entre forces armées, même si d'autres notions s'y sont aussi rattachées ${ }^{63}$.

L'ambigüité des formations impériales (et des États de l'époque moderne, qui leur ressemblent fortement, pourrait-on ajouter) ne se limite d'ailleurs pas à des questions de territoire; les frontières humaines sont tout aussi brouillées et sujettes à des redéfinitions fréquentes, renchérit l'anthropologue Ann Stoler - la prolifération de «catégories identitaires et légales» différenciées, des critères mouvants d'inclusion et d'exclusion, l'émergence de populations «ténébreuses» — accompagnent tout autant l'expansion impériale à travers les siècles. La Nouvelle-France aura pour sa part ses "nations alliées", ses «sauvages domiciliés», ses "apostats», ses «truchements», ses «esclaves», ses «Panis», ses "Outaouais», ses «coureurs des bois», ses "gens de mauvais sang»... Si cette série de désignations semble parfois plus bénigne que les classifications hiérarchiques pointilleuses et racialisées qui émanent des Antilles, et qui durcissent partout au XIX ${ }^{\mathrm{e}}$, elles sont non moins des exemples des «taxonomies turbides » qu’a produites l'expansion française dans les contrées amérindiennes à l'époque moderne. Elles accommodent tout autant la possession d'êtres humains et nourrissent elles aussi un désir de conquête ${ }^{64}$.

61. Sur la géographie discontinue, les "corridors et les enclaves», et l'absence de "rationalisation" du territoire dans les empires, voir L. Benton, In Search of Sovereignty, op. cit., 1-12. Ses réflexions fondamentales sur les liens entre le droit et la géographie impériale sont aussi élaborées dans plusieurs textes précurseurs de cette monographie, tels, «Legal Spaces of Empire: Piracy and the Origins of Ocean Regionalism », Comparative Studies in Society and History, 47,4 (2005): 700-724; «Spatial Histories of Empire», Itinerario, 30 (2006): 19-34.

62. John H. Elliott, «A Europe of Composite Monarchies», Past and Present, 137 (1992): 48-71.

63. Daniel Nordman, Frontières de France. De l'espace au territoire, XVI'-XIX ${ }^{e}$ siècles (Paris, Gallimard, Bibliothèque des histoires, 1999).

64. La plus notoire est certainement celle qu’offre le créole Martiniquais Médéric Louis-Élie Moreau de Saint-Méry, pour l'île de Saint-Domingue dans sa Description topographique, physique, civile, politique et historique de la partie française de l'isle Saint-Domingue (Philadelphie/Paris/Hambourg, Chez l'auteur/ Chez Dupont/Chez les principaux libraires, 1797-1798, 2 vol.). 
La démarche de Dominique Deslandres partage quelques-unes de ces sources d'inspiration. S'y ajoute bien sûr sa propre intuition qu'un regard transatlantique peut être fertile, intuition qui a déjà fait ses preuves dans ses nombreuses études sur les missions. Sans utiliser explicitement le langage de taxonomie ici, elle s'intéresse néanmoins à la régulation impériale de frontières humaines. Si son objectif est de formuler des hypothèses valides pour l'ensemble du Régime français, quitte à les explorer en profondeur dans des publications ultérieures, Dominique Deslandres cible aussi dans son texte un moment aussi précis que notoire: la tentative fortement théâtrale, en 1671, d'annoncer l'extension des frontières territoriales et humaines de l'autorité française dans les Grands Lacs à travers un rituel de "prise de possession" orchestré par l'officier agissant comme secrétaire de l'intendant Jean Talon, Daumont de Saint-Lusson. La grammaire rituelle déployée dans ce contexte outre-mer, nous dit Deslandres, ressemble étrangement à ce que l'on observe dans les traités consacrant des conquêtes françaises contemporaines en Europe. Au lendemain des razzias menées en Iroquoisie par l'armée de terre française, le ton est effectivement belliqueux, et l'espoir de conquête est vif. Mais au lendemain de la "prise de possession", Sault Sainte-Marie demeure un espace algonquien. Dans la Franche-Comté annexée trois ans après, en 1674, comme dans les Grands Lacs, les autorités royales françaises composent tant bien que mal avec les pouvoirs locaux ${ }^{65}$.

À bien d'autres égards, la formation de l'État français et l'expansion impériale sont en effet des processus indissociables: c'est ce que démontre aussi Helen Dewar pour les premières décennies du XVII ${ }^{e}$ siècle. Il est important de souligner le caractère innovateur de son questionnement. L'histoire coloniale a si longtemps réifié, et figé tout ce qui était «métropolitain», que ce soit au niveau de la "culture» ou des «institutions». L'histoire française a, de son côté, marginalisé les espaces non européens dans son traitement du politique. Les historiens de la Révolution haïtienne ont été parmi les premiers à rappeler l'interdépendance de certains processus qui se déroulaient des deux côtés de l'océan. Ils ont montré, entre autres, à quel point les débats entourant la «citoyenneté» dans la France révolutionnaire ont été colorés par les enjeux de l’esclavage ${ }^{66}$. Rares sont

65. Darryl Dee, Expansion and Crisis in Louis XIV's France. Franche-Comté and Absolute Monarchy, 16741715 (Buffalo, University of Rochester Press, 2009).

66. Entre autres, Malick Ghachem, The Old Regime and the Haitian Revolution (Cambridge, Cambridge University Press, 2012); David Geggus et D. B Gaspar, A Turbulent Time: The French Revolution and the Greater Caribbean (Bloomington, Indiana University Press, 1997). 
les études qui examinent des époques moins fracassantes. Helen Dewar, toutefois, réoriente notre regard vers le début du XVII siècle. Avant d'être des instruments de colonisation ou de commerce, et alors que les colonies ne sont encore que des projets presque informes, la Compagnie des CentAssociés, et les compagnies « universelles» qui l'ont précédée, sont d’abord des enjeux dans les conflits de pouvoirs internes, et dans la construction de la souveraineté dans la métropole même — souveraineté qui est loin d'être assurée en pratique, ou définie une fois pour toutes «en théorie ${ }^{67}$ ». Dans la foulée des travaux de Benton, comme de ceux des chercheurs qui renouvellent l'histoire des grandes compagnies commerciales de l'époque moderne en les assimilant à des quasi-États qui tentent d'exercer une forme de souveraineté, Helen Dewar transforme notre perspective sur des institutions que l'on croyait connaître, et sur une époque longtemps réduite à celle de "vaines tentatives ${ }^{68}$ ".

Adoptant à leur tour une échelle d'analyse transocéanique, les textes de Karin Vélez et de Timothy Pearson décloisonnent aussi des frontières, mais religieuses cette fois. Pour ces deux auteurs, l'histoire de la NouvelleFrance fait écho à des transformations qui dépassent même non seulement les confins du monde atlantique français, mais le monde atlantique tout court. Vélez, d'abord, trace des sillons surprenants du sacré et de ses appropriations transculturelles ${ }^{69}$. Chemin faisant, la chapelle de Lorette près de Québec, si familière, sera inscrite dans l'histoire globale du catholicisme: c'est par la voie du ciel, depuis Nazareth, que la sainte maison, lieu de l'annonciation à la Vierge Marie, se serait d'abord mise à voyager, pour atterrir au XIII ${ }^{e}$ siècle sur la côte adriatique de l'Italie. Sorte de relique mobile, cette chapelle portant le nom d'une simple italienne, Laureta, se réincarnera par la suite dans de nombreux sites à l'époque moderne - portée par des marins dans des ports tels Lisbonne, et surtout, par les jésuites, dans des endroits aussi dispersés que l’Allemagne, la Californie et le Canada du XVII siècle. Et si les Hurons réfugiés et leurs descendants dans la vallée du Saint Laurent semblent vouer tant d'affec-

67. James Sheehan, «The Problem of Sovereignty in European History», American Historical Review, $111(2006): 1-15$.

68. Philip J. Stern, The Company-State. Corporate Sovereignty and the Early Modern Foundations of the British Empire in India (Oxford, Oxford University Press, 2011); Edward Kavanagh, "A Company with Sovereignty and Subjects of its Own? The Case of the Hudson's Bay Company, 1670-1763 ", Canadian Journal of Law and Society/Revue Canadienne Droit et Société, 26,1 (2011): 25-50.

69. Karin Annelise Vélez, «Resolved to Fly: The Virgin of Loreto, the Jesuits \& the Miracle of Portable Catholicism in the Seventeenth-Century Atlantic World», thèse de Ph.D. en histoire, Princeton University, 2008. 
tion à leur propre version de la chapelle nomade, serait-il possible de croire que cette offrande du ciel n'est pas sans rappeler une autre chute mythique, celle de leur progénitrice iroquoienne, Femme-Ciel (Aataentsic, pour les Hurons). Pour Vélez, une telle relecture d'un chapitre de l'histoire de la toponymie du Nouveau Monde nuance l'idée qu'on peut se faire des empires plus généralement: malgré les apparences, tout ne se réduit pas à l'effacement colonialiste, ou à l'exercice unidirectionnel de pouvoir depuis des «centres» métropolitains vers des «périphéries» coloniales.

La géographie du sacré est aussi au cœur des travaux de Timothy Pearson $^{70}$. Dans ce numéro spécial, pourtant, il s'attarde plutôt à des questions de juridiction religieuse, et nous rappelle que l'histoire de la Nouvelle-France se répercute bien au-delà du traité de Paris de 1763, et bien au-delà de la Nouvelle-France même. Comme Vélez, il nous montre que pour comprendre le monde Atlantique français, il faut parfois voyager au-delà des frontières de l'empire français. Les contemporains en faisaient autant: les missions catholiques d'une Acadie conquise par la GrandeBretagne, tout comme les missions asiatiques menées par des prêtres français, forment-elles un seul corps avec le Séminaire des missions étrangères de Paris, se demandait-on? Au lendemain de la guerre de Sept Ans, après des décennies d'ambigüité dans les pratiques, un Parlement de Paris, de plus en plus défenseur de la "nation française», et fort de ses combats contre les jésuites voués à l'obéissance du pape, se lavera en quelque sorte les mains des missions, et plus précisément de ces missionnaires aux œuvres qui débordent des espaces formels de l'empire.

De Paris, bien entendu, siège du parlement ainsi que de compagnies de commerce et du Séminaire des Missions Étrangères, de l'Asie missionnaire faisant cause commune avec l'Acadie, de la Terre Sainte où la maison de la Vierge réussit son premier décollage: on voit que bien des chemins, dans le monde atlantique et au-delà, mènent à la Nouvelle-France, pour peu que l'on se donne la peine de suivre les connexions. Tout en se réservant le droit de voyager elle-même, d'une carte géographique ou prise de possession à la prochaine, cette entité coloniale nous fait voyager.

Soulignons pour terminer qu'à l'heure actuelle, elle fait également voyager toute une internationale de spécialistes. Lieu de convergence historique, la Nouvelle-France fait aussi converger les regards historiens. Ce champ d'étude est sans doute plus international que jamais, dans la

70. Timothy Pearson, «Becoming Holy in Early Canada: Performance and the Making of Holy Persons in Society and Culture», thèse de Ph.D. en histoire, Université McGill, 2008. 
foulée de la diversification de l'histoire coloniale des États-Unis autrefois abonnée aux seules Treize Colonies bien comptées ${ }^{71}$, et de la redécouverte française, voire européenne, de l'histoire coloniale ${ }^{72}$. On fait ou a pu faire l'histoire du métissage depuis l'Australie, des Acadiens depuis Florence ou Salt Lake City, de l'esclavage amérindien en Nouvelle-France depuis la Virginie, pour ne citer que quelques pôles de ce grand éparpillement ${ }^{73}$. Il s'agit là de la recomposition d'un échiquier qui s'est toujours étendu à plusieurs pays, mais qui de nos jours donne lieu à une véritable discussion internationale, du moins du point de vue des travaux cités ${ }^{74}$. En même temps, les raisons fort variées d'étudier l'histoire de la Nouvelle-France et, parfois, la tendance à ramener vers un «ici» particulier des conclusions apparemment plus vastes, témoignent de la présence de publics nationaux - et, parfois, de l'influence persistante de ces publics congelés que sont les traditions historiographiques également nationales.

Rien de plus normal, ce sont là aussi des axes, deux parmi d'autres, d'un certain pluralisme. Mais si tel est le cas, il importe de multiplier les voix. Ici, les francophones d'Amérique pourraient faire porter les leurs davantage. Autant pour enrichir l'historiographie de la Nouvelle-France élargie que celle du monde atlantique francophone ${ }^{75}$. Et pour nourrir les réflexions comparatives en cours: comme l'indiquent de récentes synthèses, l'histoire du monde atlantique français demeure somme toute moins explorée que celle de plusieurs autres Atlantiques, ce qui nuit à l'intelligence de l'ensemble ${ }^{76}$. Sans que l'on se prive nécessairement de participer aux discus-

71. Claudio Saunt, "Go West: Mapping Early American Historiography », William and Mary Quarterly, 65,4 (octobre 2008): 745-778.

72. Voir par exemple G. Havard, «L'historiographie... », loc. cit.

73. Michael A. McDonnell, «Paths Not Yet Taken, Voices Not Yet Heard: Rethinking Atlantic History", dans Ann Curthoys et Marylin Lake, dir., Connected Worlds: History in Transnational Perspective (Canberra, Australian National University Press, 2005), 45-62; Jean-François Mouhot, Les réfugiés acadiens en France 1758-1785. L'impossible intégration? (Québec, Septentrion, 2009); Christopher Hodson, The Acadian Diaspora: An Eghteenth-Century History (New York, Oxford University Press, 2012); B. Rushforth, Savage Bonds..., op. cit.

74. Par exemple, François-Joseph Ruggiu, «Une noblesse atlantique? Le second ordre français de l’Ancien au Nouveau Monde», Outre-Mers, 97,362-363 (2009): 39-63.

75. Y compris sa partie caraïbe, intimement liée à la Nouvelle-France. Un texte pionnier: Jacques Mathieu, op. cit. Philip P. Boucher, Les Nouvelles-Frances: La France en Amérique, 1500-1815 (Sillery, Septentrion, 2004), traduction de C. Broué et D. Vaugeois; Philip P. Boucher, France and the American Tropics to 1700: Tropics of Discontent? (Baltimore, Johns Hopkins University Press, 2008); James Pritchard, In Search of Empire: The French in the Americas, 1670-1730 (New York, Cambridge University Press, 2004); Shannon Lee Dawdy, «La Nouvelle-Orléans au XVIII siècle. Courants d'échange dans le monde caraibe », Annales HSS, 62,3 (2007): 663-685.

76. B. Bailyn et P. Denault, dir., Soundings..., op. cit.; J. P. Greene et P. D. Morgan, dir., Atlantic History..., op. cit. ; N. Canny et P. D. Morgan, dir., Oxford Handbook..., op. cit. Sur la géographie de l'historiographie atlantique, voir les textes issus de la journée d'études organisée par Cécile Vidal, "L’histoire atlantique 
sions se déroulant uniquement en anglais - le plus souvent états-unien -, le commun objet de recherche crée une communauté interprétative internationale caractérisée par une connaissance au moins passive du français. Outre les retrouvailles dans les notes infrapaginales et dans les colloques - qu'on peut espérer de plus en plus virtuels à mesure que le carbone s'accumule dans l'atmosphère terrestre -, cela suppose un certain effort pour créer, à travers les recensions et les notes critiques, une chambre de résonance critique et francophone. Du point de vue québécois, cette Revue et le congrès annuel de l'Institut, également «de l'Amérique française», sont les lieux tout désignés pour ces échanges. Ces derniers pourraient bien sûr aussi porter sur des phases moins anciennes de cette histoire transfrontalière, voire transocéanique. Que ce numéro thématique suscite des vocations... 